\section{Pre-hospital rapid sequence induction: Factual inaccuracies?}

To the Editor: The article on pre-hospital rapid sequence induction (RSI) in the September issue of the $S A M J^{[1]}$ makes a valuable contribution to our understanding of RSI in this environment in South Africa (SA). However, there are some factual inaccuracies in the article regarding the educational background of those performing the procedure, which I would like to clarify.

In SA, emergency care personnel are registered with the Health Professions Council of South Africa (HPCSA) in different categories depending on the qualification that they hold. Although the term 'paramedic' is used in a very general way in the international literature, in SA it has a specific meaning in relation to a specific category of registration. Currently, RSI does not exist in the scope of practice of emergency care personnel registered in the category of 'paramedic', but only in the registration category of emergency care practitioner (ECP). ${ }^{[2]}$ This distinction is of some importance when considering the educational background of those practicing RSI, as ECPs hold a four-year degree in emergency medical care (EMC).

On page 633, and again on page 636, the authors describe RSI training as being a 'two day university course' and that they consider this to be insufficient. ${ }^{[1]}$ I agree that two days of training on RSI is insufficient. However, this is not an accurate description of current RSI education at all institutions offering the EMC degree programme. The 'two day university course' was offered once in 2009 by one institution for those who already held an EMC degree, as a way of incorporating RSI (which was newly-approved at the time) into their clinical practice. The important point is that it was a 'once-off' arrangement intended to update those who qualified with an EMC degree before RSI was approved for ECP scope of practice; it has not been the approach followed for undergraduate students since 2010 .

At the University of Johannesburg (UJ), RSI has been incorporated into an educational strategy on airway management which spans four years. Our approach in designing and implementing RSI education since 2010 has involved advice, input and involvement from specialists in both anaesthesiology and emergency medicine. Assessments include a mix of theory, objective structured clinical evaluations, simulations and assessments carried out in the emergency centre and pre-hospital environments.

While we would like all of our students to have clinical experience in RSI before qualifying as independent practitioners, there is a very real problem of access to critical procedures. The healthcare system caters for a large number of students from a variety of fields and institutions. All compete for clinical experience in a small number of facilities that have suitable levels of clinical supervision. Given these constraints, we are currently implementing the approach outlined in the position statement referred to by the authors in their introduction. ${ }^{[3]}$ Considering the above, our qualifying students have done more than a 'two day course. ${ }^{\text {[1] }}$ Likewise, the authors' assertion that 'In SA, trainee paramedics are required to perform 3 clinical RSI scenarios successfully to meet graduation requirements ${ }^{[1]}$ is not true.

The authors state on page 633 that RSI was performed according to a standard operating procedure (SOP) which included the use of thiopentone as an induction agent. ${ }^{[1]}$ It is unclear whether the SOP was used only within the private emergency medical service (EMS) from which data for the study were drawn, or whether the authors are referring to a protocol for RSI published by the HPCSA. Either way, thiopentone has never been approved for use as an induction agent for RSI by ECPs. ${ }^{[2]}$ If the SOP referred to was an 'in-house' one, then the inclusion of thiopentone as an option for induction is in conflict with the HPCSA's ruling on approved induction agents for ECP use.
Lastly, the authors highlight a critical limitation with the way that data on RSI are currently recorded. To address this, the UJ has developed a standardised dataset (based on a recommended dataset for the reporting of airway interventions from the USA) ${ }^{[4]}$ and an online RSI registry that we hope will be implemented soon (we are awaiting approval of an agreement specifying the terms of operation and joint responsibilities from the HPCSA, with whom all ECPs are registered).

\section{Stein}

Department of Emergency Medical Care, University of Johannesburg, South Africa cstein@uj.ac.za

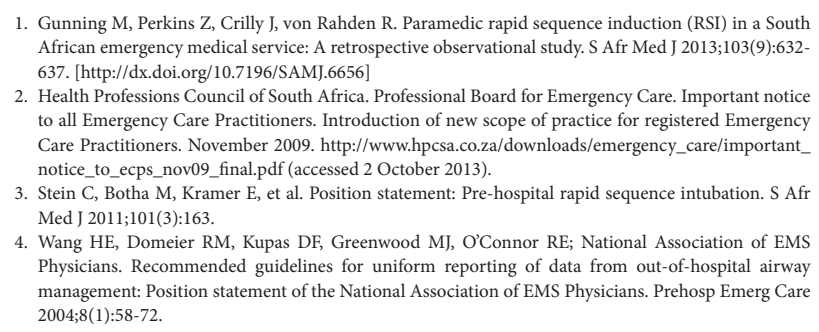

1. Gunning M, Perkins Z, Crilly J, von Rahden R. Paramedic rapid sequence induction (RSI) in a South African emergency medical service: A retrospective observational study. S Afr Med J 2013;103(9):632637. [http://dx.doi.org/10.7196/SAMJ.6656]

2. Health Professions Council of South Africa. Professional Board for Emergency Care. Important notice to all Emergency Care Practitioners. Introduction of new scope of practice for registered Emergency Care Practitioners. November 2009. http://www.hpcsa.co.za/downloads/emergency_care/important_ notice_to_ecps_nov09_final.pdf (accessed 2 October 2013).

3. Stein C, Botha M, Kramer E, et al. Position statement: Pre-hospital rapid sequence intubation. S Afr Med J 2011;101(3):163.

4. Wang HE, Domeier RM, Kupas DF, Greenwood MJ, O'Connor RE; National Association of EMS Physicians. Recommended guidelines for uniform reporting of data from out-of-hospital airway management: Position statement of the National Association of EMS Physicians. Prehosp Emerg Care
mation 2004;8(1):58-72.

Gunning responds: Paramedic RSI remains controversial, as it is a high-risk procedure where safer alternatives may exist.

RSI-qualified 'paramedic' was used in our manuscript as it is a more widely used and understood definition in the international literature than the term 'ECP': the training and scope of practice of whom are not uniform, ${ }^{[1]}$ and may not include RSI outside SA.

RSI competence is related to prior operator experience, but this was unknown in our cohort of the first paramedic RSIs performed by this emergency medical service. We reported the training that this initial cohort of paramedics received, but applaud the ongoing improvements in the training programme. We remain concerned, however, about the limited access to critical procedures with suitable supervision. Knowledge is important, but ensuring competence is imperative before unsupervised provision of a high-risk to benefit ratio intervention can be endorsed.

The Advanced Airway Management and RSI SOP (valid July 2011 to June 2012), provided by the private EMS for the purpose of this study, included the use of thiopentone. This SOP was to be read in conjunction with the HPCSA's guidance, and as such, paramedics were not authorised to administer thiopentone in the absence of a medical officer, as demonstrated in Table 3 of our manuscript.

Our manuscript highlighted that poor documentation negatively impacted on the quality of the audit. We are encouraged by attempts to improve data capture, and would like to point out that an agreed standardised Utstein Style template already exists for this purpose..$^{[2]}$

To conclude, we urge the establishments teaching this intervention to fund and co-ordinate a robust study (randomised controlled trial) to answer the actual underlying question 'does paramedic RSI benefit patients in SA?'

\section{Gunning}

Department of Anaesthetics, Inkosi Albert Luthuli Hospital, Durban, South Africa mattgunning@rcsed.ac.uk

\footnotetext{
1. Woolard M. The role of the paramedic practitioner in the UK.JEPHC 2006;4(1):1-8.

2. Sollid SJ, Lockey D, Lossius HM; Pre-hospital advanced airway management expert group. A consensusbased template for uniform reporting of data from pre-hospital advanced airway management. Scand J Trauma Resusc Emerg Med 2009;17:58. [http://dx.doi.org/10.1186/1757-7241-17-58]
} 